\title{
Proyecciones de las relaciones Internacionales de la Sociedad Chilena de Enfermedades Respiratorias
}

\section{Projections of Chilean Respiratory Society international relationships}

La formación continua es uno de los objetivos estratégicos de la Sociedad Chilena de Enfermedades Respiratorias (SER), dirigida tanto a sus socios, como a aquellos profesionales de la salud que participan en la atención de pacientes con enfermedades respiratorias. Pretendemos mejorar no sólo los conocimientos, sino también las competencias y habilidades en el desempeño profesional diario de médicos especialistas y no especialistas, y de profesionales fundamentales en la atención integral de nuestros pacientes, tales como kinesiólogos, enfermeras, tecnólogos-médicos o químicos farmacéuticos.

En el intento de conseguir en mejor forma estos importantes objetivos, la SER ha establecido asociaciones con otras sociedades cientificas del país y del extranjero. Debe destacarse el reciente trabajo conjunto con la Sociedad Médica de Santiago y la Sociedad Chilena de Neumología Pediátrica y el nuevo acercamiento con la Sociedad Chilena de Alergia e Inmunología, recordando tiempos anteriores en que se efectuó un fructífero trabajo conjunto.

A nivel internacional tenemos desde hace años, cordiales relaciones con la Sociedad Respiratoria Europea (ERS), con la Sociedad Americana de Tórax (ATS), con la Asociación Latinoamericana de Tórax (ALAT) y en el año 2012 firmamos un convenio de colaboración con la Sociedad Española de Neumología y Cirugía de Tórax (SEPAR).

Surge aquí la legítima pregunta: ¿En qué forma debemos orientar las relaciones internacionales de la SER y cuáles podrían ser sus beneficios y proyecciones? A juicio de quien escribe este editorial, las relaciones internacionales de la SER deben potenciarse, lo que permitiría recibir beneficios de aquellas sociedades más organizadas y mejor estructuradas, sin dejar de reconocer que en la SER tenemos una trayectoria de trabajo y funcionamiento, con importantes aportes cientificos de nuestros socios, que legitimamente debemos estar orgullosos en presentarlos y difundirlos.

A corto plazo, algunas de las formas que parecen beneficiosas a desarrollar son:

1. Colaboración en los congresos de las sociedades con las que ya hemos formalizado relaciones. Este año 2013, iniciamos esta modalidad con la SEPAR, efectuando un simposio conjunto, en el cual expondrán ideas socios de ambas entidades, pretendiéndose mantener este esquema de intercambio de invitados tanto en congresos de la SER, como de la SEPAR. Debemos aumentar nuestra influencia a nivel de ALAT, de modo que logremos tener a futuro al menos un simposio conjunto en nuestro congreso y aumentar nuestra influencia en los congresos ALAT. Un poco más lejano aparece la opción de influir en congresos organizados por sociedades como la ERS o la ATS. Sin embargo, el reciente simposio ERS-ALAT realizado en español en el último congreso de la ERS, plantea una nueva forma de relación entre instituciones europeas y latinoamericanas.

2. Investigación Clínica entre sociedades. Desarrollar este tema parece ser extraordinariamente interesante. Nuestra sociedad debe transformarse en un punto de unión y lograr la integración de nuestros socios investigadores con equipos de investigación de las sociedades ya señaladas. Los proyectos que requieren un número importante de pacientes como lo son entre otros, los proyectos multicéntricos en epidemiología clínica, los estudios en enfermedades poco frecuentes y la evaluación de modificaciones de conductas en aplicación de guías clínicas, se verán favorecidos en su desarrollo por estas alianzas y al mismo tiempo, permitirán estudiar las diferenciales regionales que podemos tener en cada uno de ellos. Para lograr estos objetivos se requerirá el firme compromiso de los miembros de las sociedades involucradas. 
3. Intercambio de docentes e investigadores. Las relaciones logradas entre estas sociedades con fines comunes, debería permitirnos lograr el financiamiento para que los miembros de las sociedades cientificas implicadas puedan hacer estadias programadas en diferentes grupos de trabajo con fines de capacitación y perfeccionamiento o de investigación. Este intercambio de experiencias presenciales han demostrado que modifican favorablemente las competencias de la persona que es beneficiada con este intercambio y el grupo que lo recibe, logrando además un mayor acercamiento entre las instituciones que lograron este objetivo. Constituirá en la práctica un estímulo para lograr un mayor grado de acercamiento entre la sociedad y sus miembros.

Concluyendo estas breves reflexiones, se entiende que el estímulo de las relaciones internacionales de la SER con las sociedades descritas u otras que puedan surgir, constituyen un desafío, pero al mismo tiempo una potente área de desarrollo para la SER y sus miembros.

Dr. Mario Calvo G.

Vicepresidente,

Sociedad Chilena de Enfermedades Respiratorias 